\title{
Acupunctura na dor lombar: há evidência?
}

João Monteiro,* Elisa Ribeiro**

\section{RESUMO}

Objectivos: Rever a evidência disponível sobre a eficácia da acupunctura no tratamento da dor lombar.

Fontes de Dados: MEDLINE, National Guideline Clearinghouse, Guidelines Finder, Cochrane Library, DARE, Bandolier, UpToDate e Index de Revistas Médicas Portuguesas.

Métodos de Revisão: Realizada pesquisa bibliográfica, em Abril e Maio de 2009, utilizando os termos MeSH acupuncture e low back pain e os termos DeCS acupuntura e dor lombar. Limitou-se a pesquisa a artigos publicados entre Janeiro de 2000 e Maio de 2009, em inglês, espanhol e português. Os estudos foram classificados usando a escala Strength of Recommendation Taxonomy (SORT).

Resultados: Obtiveram-se 154 artigos, tendo 14 cumprido os critérios de inclusão (6 revisões sistemáticas, 2 meta-análises, 3 ensaios controlados aleatorizados e 3 normas de orientação clínica). As normas de orientação clínica referem que a acupunctura pode ser útil no tratamento da dor lombar. Relativamente à dor lombar crónica inespecífica, as meta-análises, revisões sistemáticas e ensaios demonstraram que a acupunctura é mais eficaz do que a ausência de tratamento e que a associação daquela com a terapêutica convencional é mais eficaz do que a terapêutica convencional isoladamente. Os dados relativos à comparação da acupunctura com placebo e com outros tratamentos não demonstraram que a acupunctura fosse mais eficaz. Na dor lombar aguda os dados são inconclusivos.

Conclusões: A acupunctura tem eficácia no tratamento da dor lombar crónica inespecífica, isoladamente ou como co-adjuvante (SORT A); não ficou demonstrado que fosse superior a outros tratamentos. Não foi demonstrada eficácia no tratamento da dor lombar aguda. É importante a realização de estudos de custo-benefício, informação acerca dos terapeutas e aguardar normas mais específicas.

Palavras-chave: Dor Lombar; Acupunctura.

\section{INTRODUÇÃO}

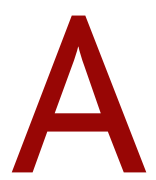
dor lombar é um importante problema de saúde nos países desenvolvidos e é uma das principais razões pelas quais adultos procuram tratamento médico. Aproximadamente 70 a $85 \%$ dos adultos sofrerão de dor lombar em algum momento da sua vida ${ }^{1,2}$ e mais do que $50 \%$ sofrem de dor lombar anualmente. ${ }^{3}$

Nos Estados Unidos da América é a causa mais frequente de inactividade em indivíduos com menos de 45 anos de idade e a segunda causa mais frequente de consulta médica. ${ }^{4}$ Em Inglaterra, à semelhança de outros países, é uma das patologias mais dispendiosas,

*Interno de Medicina Geral e Familiar, Unidade de Saúde Familiar das Ondas, ACES Porto V - Póvoa de Varzim/Vila do Conde

**Interna de Medicina Geral e Familiar, Unidade de Saúde Familiar Santa Clara,ACES Porto V - Póvoa de Varzim/Vila do Conde não só por custos directos e indirectos relacionados com a saúde, mas também pelo absentismo e perda de produção que causa. ${ }^{5}$ Associado ao impacto sócio económico há ainda o impacto psicossocial, que tornam a dor lombar uma condição particularmente importante e com necessidade de tratamento eficaz. ${ }^{2}$

Apesar de 90\% dos episódios agudos de dor lombar resolver no espaço de seis semanas, cerca de $7 \%$ dos doentes irão desenvolver dor lombar crónica. ${ }^{6}$ Assim, doentes com dor lombar aguda geralmente melhoram no que diz respeito à dor e incapacidade funcional, regressando ao trabalho dentro de um mês. Até aos três meses, adicionais e ligeiras melhorias ocorrem. A partir desta data a dor e a incapacidade permanecem quase constantes e a maioria dos doentes terá uma recorrência nos 12 meses seguintes. ${ }^{7}$ A dor lombar inespecífica representa a grande maioria de casos de dor lombar. ${ }^{5}$ 
Um tratamento eficaz desta patologia é importante, não só do ponto de vista da qualidade de vida dos doentes, mas também na perspectiva social. ${ }^{2}$ De forma crescente, doentes têm procurado terapias médicas alternativas e complementares para adicionar aos cuidados médicos convencionais, no sentido de aliviar a sintomatologia da dor lombar. ${ }^{8}$ No entanto, a sua eficácia e relação custo-benefício, individualmente ou em associação com outras abordagens terapêuticas, permanecem incertas. ${ }^{8}$

A acupunctura tem ganho popularidade no mundo ocidental e o seu uso tem-se disseminado por diversos países em todo o mundo. ${ }^{9}$ Actualmente, é uma intervenção largamente aceite para o tratamento de uma variedade de patologias, muitas das quais associadas à dor. $^{10}$

A acupunctura é uma das mais antigas abordagens terapêuticas e tem as suas raízes na medicina tradicional chinesa. ${ }^{7}$ Consiste na estimulação de pontos corporais específicos ${ }^{11}$ e o seu mecanismo de acção ainda não é claro, havendo diversas teorias.

A analgesia causada pela acupunctura tem uma base fisiológica, anatómica e neuroquímica, originada nos impulsos da contracção muscular local sob o ponto de acupunctura. É essencialmente uma manifestação de processos integrativos a diferentes níveis do sistema nervoso central entre impulsos aferentes das regiões dolorosas e impulsos dos pontos de acupunctura. Mecanismos segmentares ao nível da espinhal medula contribuem para a relativa especificidade funcional dos pontos de acupunctura, usando as vias nervosas presentes nos funículos antero-laterais. Há, ainda, vários neurotransmissores envolvidos, nomeadamente peptídeos opióides, glutamato, 5-hidroxitriptamina e noradrenalina. ${ }^{12}$

Em Inglaterra, a acupunctura é frequentemente usada quer no Serviço Nacional de Saúde, quer no âmbito da medicina privada. Aproximadamente $7 \%$ da população adulta já fez pelo menos um tratamento com acupunctura.
A dor lombar é uma das principais indicações da acupunctura $^{13}$, sendo o principal motivo de consulta dos profissionais de saúde que a praticam e representando uma em cada sete consultas. ${ }^{3}$ Contudo, o seu valor no tratamento da dor lombar é ainda controverso. ${ }^{1}$

Por este motivo, realizou-se o presente estudo com o objectivo de rever a evidência disponível sobre a eficácia da acupunctura no tratamento da dor lombar nos adultos.

\section{MÉTODOS}

Foi realizada uma pesquisa bibliográfica em Abril e Maio de 2009 na MEDLINE, National Guideline Clearinghouse, Guidelines Finder, Cochrane Library, DARE, Bandolier, UpToDate e Index de Revistas Médicas Portuguesas, utilizando os termos MeSH acupuncture e low back paine os termos DeCS acupunctura e dor lombar. Limitou-se a pesquisa a artigos publicados entre Janeiro de 2000 e Maio de 2009, em inglês, espanhol e português.

Para avaliar a qualidade dos estudos e a força de recomendação, foi utilizada a escala Strength of Recommendation Taxonomy (SORT) da American Family Physician (AFP). ${ }^{1}$

Define-se dor lombar aguda como tendo menos de 12 semanas de evolução e dor lombar crónica como tendo mais de 12 semanas de evolução. A dor lombar específica é uma dor lombar atribuível a um problema estrutural, como núcleo pulposo herniado, fractura, artrite, tumor ou infecção; na dor lombar inespecífica há queixas vagas e difusas de dor, intermitentes, recorrentes e com exame neurológico normal, sendo essencialmente um diagnóstico de exclusão. ${ }^{2}$

A acupunctura consiste na estimulação de pontos anatómicos cutâneos e musculares com agulhas, havendo vários estilos: chinesa, japonesa e ocidental. A acupunctura-placebo é uma intervenção com a intenção de fazer crer aos pacientes que estão a ser tratados com acupunctura; normalmente envolve a simulação de inserção, a inserção apenas superficial ou a inserção das agulhas de acupunctura em locais anatómicos diferen-

\footnotetext{
a Segundo a escala SORT, a qualidade do estudo está subdividida em três níveis de evidência (nível de evidência 1: estudos de boa qualidade, evidência orientada para o doente; nível de evidência 2: estudos de qualidade limitada, evidência orientada para o doente; e nível de evidência 3: outra evidência) e a força de recomendação em três graus (força de recomendação A: consistente, boa qualidade, evidência orientada para o doente; força de recomendação B: inconsistente ou qualidade limitada, evidência orientada para o doente; e força de recomendação C: consensos, evidência orientada para a doença) ${ }^{14}$
} 


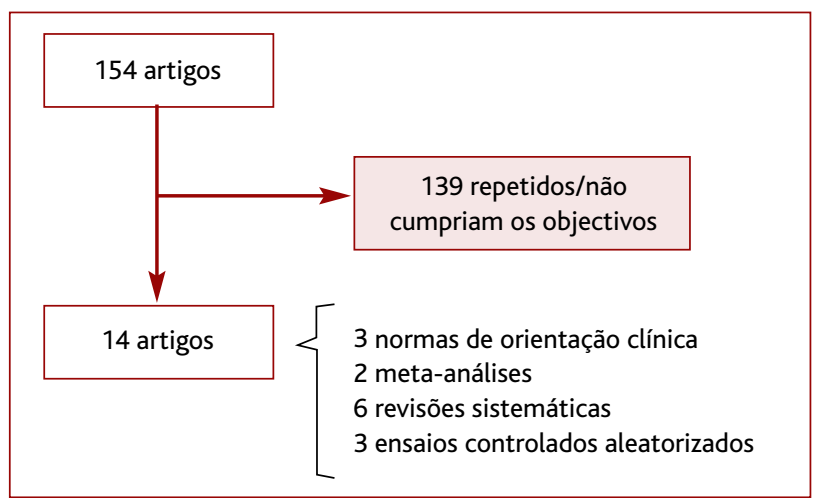

Figura 1. Metodologia do estudo. tes dos indicados pelos diferentes tipos de acupunctura para cada patologia em particular. Os outros tratamentos presentes nos diversos estudos correspondem a massagem, exercício, terapêutica comportamental, manipulação espinhal, electroestimulação nervosa transcutânea e anti-inflamatórios não esteróides. ${ }^{4,15}$

Foi avaliado o efeito da acupunctura na dor lombar (aguda ou crónica) em adultos, comparando-a com a ausência de tratamento, com placebo, com outros tratamentos e usando-a em associação com outras terapêuticas. Os outcomes estudados foram a dor e o aumento da capacidade funcional.

QUADRO I. Resultados (Normas De Orientação Clínica)

Referência Recomendação FR

\begin{tabular}{l|l|l}
\hline $\begin{array}{l}\text { Council of Acupuncture and } \\
\text { Oriental Medicine Associates } 2004^{16}\end{array}$ & O uso da acupunctura é adequado no tratamento da dor lombar \\
\hline Amerian College
\end{tabular}

American College of Physicians and No tratamento da dor lombar, a acupunctura é opção quando the American Pain Society $2007^{17}$ não há melhoria com terapêutica farmacológica, em casos de dor sub-aguda ou crónica

NICE Clinical Guideline $2009^{18}$

A acupunctura pode ajudar a diminuir a dor lombar

\begin{tabular}{|l} 
B \\
\hline
\end{tabular}

Legenda: FR - Força de recomendação (Força de recomendação atribuída pelos autores das normas de orientação clínica)

\section{QUADRO II. Resultados (Ensaios Controlados Aleatorizados)}

Referência

Motohiro Inoue et al

Acupuncture Medicine, $2006^{19}$

Kazunori Itoh, et al Acupuncture Medicine, $2006^{20}$

S. Kennedy et al Complement Ther Med, $2008^{21}$

\section{Objectivo/Metodologia}

Efeito da acupunctura na dor lombar inespecífica $\mathrm{N}=31$

Acupunctura versus Placebo

Outcomes: dor (EAV) e função (Teste de Schober) Endpoint: Imediato

Avaliar a eficácia da acupunctura na dor lombar crónica

$\mathrm{N}=26$, com mais de 65 anos

Acupunctura versus Placebo

Outcomes: dor (EAV) e função (Roland - Morris

Disability Questionnaire)

Endpoint: Imediato

Eficácia da acupunctura na dor lombar aguda

$\mathrm{N}=48$, entre os 18 e os 70 anos

Acupunctura versus Placebo

Outcomes 1os: dor (EAV) e função (Roland -

Morris Disability Questionnaire)

Endpoints: Imediato e aos 3 meses

\begin{tabular}{l|l} 
Resultados & NE \\
$\begin{array}{l}\text { Superioridade estatisticamente } \\
\text { significativa da acupunctura }\end{array}$ & 2 \\
\hline $\begin{array}{l}\text { Superioridade estatisticamente } \\
\text { significativa da acupunctura }\end{array}$ & 2 \\
\hline $\begin{array}{l}\text { Sem diferença estatisticamente } \\
\text { significativa, excepto para os } \\
\text { outcomes } 2^{\text {os }} \text { (toma de } \\
\text { analgésicos e pior dor sentida), } \\
\text { com melhores resultados no grupo } \\
\text { tratado com acupunctura }\end{array}$ & 2 \\
\hline
\end{tabular}




\section{RESULTADOS}

Dos 154 artigos encontrados, 140 foram excluídos por se encontrarem repetidos, por haver discordância com o objectivo da revisão ou por não cumprirem os critérios de inclusão (Figura 1). Obtiveram-se, desta forma, 14 artigos: três normas de orientação clínica (NOC), duas meta-análises (MA), seis revisões sistemáticas (RS) e três ensaios controlados aleatorizados (ECA) - Quadros I, II, III e IV.

As NOC obtidas na pesquisa recomendam o uso da acupunctura no tratamento da dor lombar ${ }^{16,18}$, havendo a indicação do seu uso, de acordo com a NOC publicada na American College of Physicians and the American Pain Society, ${ }^{17}$ quando não há melhoria com terapêutica farmacológica em casos de dor lombar subaguda ou crónica. As MA de Eric Manheimer, MS et al ${ }^{4}$ e de Furlan AD et al tinham como objectivo o estudo da eficácia da acupunctura no tratamento da dor lom- bar. Relativamente à dor lombar aguda os dados ou foram inconclusivos ou foi demonstrado claramente não haver eficácia da acupunctura. Na dor lombar crónica, a acupunctura demonstrou ser mais eficaz do que a ausência de tratamento e do que o placebo, não se revelando superior comparativamente com outros tratamentos. Demonstrou ainda, na MA de Furlan AD et al, ${ }^{7}$ que o seu uso em associação com abordagens terapêuticas convencionais era mais eficaz do que o uso desses tratamentos isoladamente.

Relativamente às seis RS, nos estudos de Van Tulder $M W$ et $a l^{25} e$ de Roger Chou et $a l^{23}$ constatou-se que na dor lombar aguda não há dados conclusivos ou não há evidência de eficácia da acupunctura comparativamente com placebo ou outros tratamentos. Na dor lombar crónica, a acupunctura demonstrou ser eficaz quando comparada com a ausência de tratamento $0^{5,15,23-}$ ${ }^{25}$ ou quando comparada com placebo. ${ }^{15,22,23,25}$ Pelo con-

\section{QUADRO III. Resultados (Meta-Análises)}

\section{Referência}

Eric Manheimer, MS et al

Ann Intern Med, $2005^{4}$

\section{Objectivo/Metodologia}

Eficácia da acupunctura na dor lombar

33 ECA (2300 doentes)
Furlan $A D$ et al

Cochrane, $2005^{7}$
Eficácia da acupunctura no tratamento da dor lombar inespecífica 35 ECA (2861 doentes): DLC (32 ECA) e DLA (3 ECA)

\begin{tabular}{|l|l} 
Resultados & NE \\
\hline Dor lombar aguda: dados inconclusivos & 1 \\
Dor lombar crónica: & \\
AC versus Sem tratamento - AC mais eficaz & \\
na dor e função (curto-prazo) e dor & \\
(longo-prazo) & \\
AC versus Placebo - AC mais eficaz na dor a & \\
curto e longo-prazo & \\
AC versus Outros tratamentos - não há & \\
evidência da AC ser mais eficaz & \\
Conclusão: A acupunctura alivia eficazmente & \\
a dor lombar crónica. & \\
\hline Dor lombar aguda: Sem evidência & 1 \\
Dor lombar crónica: & \\
AC versus Sem tratamento - AC mais eficaz & \\
(no imediato e curto-prazo) & \\
AC versus Placebo - AC mais eficaz & \\
(no imediato e curto-prazo) & \\
AC versus Outros tratamentos - AC não tem & \\
maior eficácia & \\
Terapêutica convencional versus Associação & \\
- a associação é mais eficaz & \\
Conclusão: A acupunctura é útil isoladamente/ & \\
/adjuvante na dor lombar crónica. &
\end{tabular}

Legenda: NE - Nível de evidência; AC - Acupunctura; DLC - Dor lombar crónica; DLA - Dor lombar aguda; ECA - Ensaio controlado aleatorizado 


\begin{tabular}{|c|c|c|c|}
\hline Referência & Objectivo/Metodologia & Resultados & $\mathrm{NE}$ \\
\hline $\begin{array}{l}\text { David J. Mayer } \\
\text { Annu. Rev. Med, } 2000^{22}\end{array}$ & $\begin{array}{l}\text { Avaliar o efeito da acupunctura. } \\
\text { DLC - } 10 \text { ECA }\end{array}$ & $\begin{array}{l}\text { Dor lombar crónica: } \\
\text { AC versus Placebo - Há alguma evidência da AC ser mais } \\
\text { eficaz } \\
\text { AC versus Outros tratamentos - Sem evidência de maior } \\
\text { eficácia da AC }\end{array}$ & 2 \\
\hline $\begin{array}{l}\text { Van Tulder MW et al } \\
\text { Best Pract Res Clin } \\
\text { Rheumatol, } 2005^{25}\end{array}$ & $\begin{array}{l}\text { Avaliar o efeito de terapias } \\
\text { complementares no tratamento } \\
\text { da dor lombar. } \\
\text { Acupunctura - } 20 \text { ECA } \\
\text { ( } 909 \text { doentes): } \\
\text { DLA ( } 2 \text { ECA) e DLC ( } 18 \text { ECA) }\end{array}$ & $\begin{array}{l}\text { Dor lombar aguda: } \\
\text { AC versus Placebo e AC versus AINE: sem evidência de maior } \\
\text { eficácia da AC } \\
\text { Dor lombar crónica: } \\
\text { AC versus Sem tratamento - AC mais eficaz a curto prazo; } \\
\text { evidência limitada de ser mais eficaz a médio-prazo para «dor» } \\
\text { AC versus Placebo - eficácia da AC para «dor» a curto-prazo } \\
\text { AC versus Outros tratamentos - não há evidência da AC ser } \\
\text { mais eficaz } \\
\text { Terapêutica convencional versus Associação - eficácia na } \\
\text { associação (no imediato, curto e médio-prazo) }\end{array}$ & 1 \\
\hline $\begin{array}{l}\text { A.Keller et al } \\
\text { Spine, } 2007^{15}\end{array}$ & $\begin{array}{l}\text { Eficácia de tratamentos } \\
\text { não-cirúrgicos na dor lombar } \\
\text { Acupunctura - } 7 \text { ECA (528 } \\
\text { doentes com DLC) }\end{array}$ & $\begin{array}{l}\text { Dor lombar crónica: } \\
\text { AC versus Placebo e AC versus Sem tratamento - evidência } \\
\text { da AC ser eficaz a curto-prazo (< } 6 \text { semanas) }\end{array}$ & 1 \\
\hline $\begin{array}{l}\text { Roger Chou et al } \\
\text { Ann Intern Med, } 2007^{23}\end{array}$ & $\begin{array}{l}\text { Eficácia de terapêuticas } \\
\text { não-farmacológicas na dor } \\
\text { lombar aguda e crónica } \\
\text { Acupunctura: } 3 \text { RS (com } 51 \text { ECA, } \\
\text { cujas amostras variaram entre } \\
17 \text { e } 492 \text { doentes) e } 3 \text { ECA } \\
\text { (3380 doentes) }\end{array}$ & $\begin{array}{l}\text { Dor lombar aguda: } \\
\text { AC versus Placebo e AC versus Outros tratamentos - dados } \\
\text { inconclusivos } \\
\text { Dor lombar crónica: } \\
\text { AC versus Sem tratamento - AC moderadamente eficaz às } \\
6 \mathrm{~S}, 12 \mathrm{~S} \text { e > } 6 \mathrm{M} \\
\text { AC versus Placebo - AC moderadamente eficaz para «dor» a } \\
\text { curto prazo; sem evidência a longo prazo } \\
\text { AC versus Outros tratamentos - AC sem evidência de maior } \\
\text { eficácia } \\
\text { Terapêutica convencional versus Associação - maior eficácia } \\
\text { da associação }\end{array}$ & 1 \\
\hline $\begin{array}{l}\text { Carlo Ammendolia et al } \\
\text { Spine, } 2008^{24}\end{array}$ & $\begin{array}{l}\text { Eficácia da acupunctura na dor } \\
\text { lombar crónica } \\
6 \text { RS e } 22 \text { ECA ( } 4998 \text { doentes) }\end{array}$ & $\begin{array}{l}\text { Dor lombar crónica: } \\
\text { AC versus Sem tratamento - AC mais eficaz no imediato e a } \\
\text { curto prazo } \\
\text { AC versus Placebo - dados inconclusivos } \\
\text { AC versus Outros tratamentos - AC sem evidência de ser } \\
\text { mais eficaz } \\
\text { Terapêutica convencional versus Associação - maior eficácia } \\
\text { da associação } \\
\text { Conclusão: Há alguma evidência para o uso da acupunctura } \\
\text { no tratamento da dor lombar crónica. }\end{array}$ & 1 \\
\hline $\begin{array}{l}\text { Yuan J. et al } \\
\text { Spine, } 2008^{5}\end{array}$ & $\begin{array}{l}\text { Eficácia da acupunctura na dor } \\
\text { lombar inespecífica } \\
23 \text { ECA ( } 6359 \text { doentes): } \\
\text { DLC - } 19 \\
\text { DLSA - } 1 \\
\text { DLC/SA - } 3\end{array}$ & $\begin{array}{l}\text { AC versus Sem tratamento - evidência moderada de que a } \\
\text { AC é mais eficaz } \\
\text { AC versus Placebo - sem maior eficácia da AC } \\
\text { AC versus Outros tratamentos - resultados não conclusivos } \\
\text { Terapêutica convencional versus Associação - associação é } \\
\text { mais eficaz } \\
\text { Conclusão: Acupunctura versus não tratar ou como } \\
\text { complemento de terapêutica convencional deve ser incluída } \\
\text { no tratamento da dor lombar. Individualmente, não é } \\
\text { diferente do placebo. }\end{array}$ & 1 \\
\hline
\end{tabular}

Legenda: NE - Nível de evidência; AC - Acupunctura; DLC - Dor lombar crónica; DLA - Dor lombar aguda; DLSA - Dor lombar subaguda; RS - Revisão sistemática; ECA - Ensaio controlado aleatorizado; AINE - Anti-inflamatório não-esteróide; S - semanas; M - meses 
trário, nas RS de Yuan J. et a $\rrbracket^{5}$ e de Carlo Ammendolia et $a l^{4}$ os dados comparativos com placebo ou se revelaram inconclusivos ou, por outro lado, demonstraram ausência de maior eficácia por parte da acupunctura. Nalgumas RS também foi estabelecida a comparação da acupunctura com outros tratamentos, tendo-se concluído que a acupunctura não é mais eficaz do que aqueles. ${ }^{5,22-25}$ Outro grupo de estudo abordado nas RS foi a terapêutica convencional comparada com a associação da acupunctura à terapêutica convencional, tendose determinado a existência de maior eficácia da associação. .,23-25 $^{2}$

Os ECA de Motohiro Inoue et $a l^{19}$ e de Kazunori Itoh, et $a l^{20}$ demonstraram que a acupunctura é mais eficaz do que o placebo em casos de dor lombar inespecífica e crónica, sendo os outcomes primários avaliados nesses estudos a dor (através da Escala Analógica Visual) e a função da coluna lombar (através do Teste de Schober ou do Questionário de Roland-Morris). No ECA de S. Kennedy et $\boldsymbol{l}^{11}$ não houve diferença estatisticamente significativa entre a acupunctura e o placebo, relativamente aos outcomes primários, em doentes com dor lombar aguda; houve diferença estatisticamente significativa apenas nos outcomes secundários avaliados, nomeadamente na toma de analgésicos e na pior dor sentida pelo doente, com melhores resultados no grupo tratado com acupunctura.

\section{CONCLUSÕES}

Os três ECA incluídos neste estudo visavam essencialmente a comparação da Acupunctura com uma técnica-placebo, de modo a perceber se há algum efeito específico da acupunctura ou se esta funciona fundamentalmente à custa de um importante efeito placebo. Dois dos estudos revelaram superioridade da acupunctura, ao contrário do ECA de S. Kennedy et al. ${ }^{21}$ No entanto, relativamente aos outcomes secundários, este estudo revelou existir diferença estatisticamente significativa na medicação que o doente necessitou no endpoint imediato e na pior dor sentida pelo doente no endpoint três meses, com melhores resultados para o grupo tratado com acupunctura. A metodologia de todos estes ECA apresenta importantes limitações no que diz respeito ao tamanho das amostras e à ausência de seguimento a longo-prazo. $\mathrm{O}$ facto de ter havido uma taxa de drop-out superior a $20 \%$ no estudo de Kazuno- ri Itoh et al $^{20}$ foi também uma limitação importante. As NOC são consensuais no que diz respeito à utilidade da acupunctura no tratamento da dor lombar, havendo apenas numa a especificação de uso em casos de dor sub-aguda ou crónica. No entanto, não há referência relativamente ao número de sessões, duração do tratamento ou técnica de acupunctura mais apropriada.

As MA e RS demonstram que os dados relativamente à eficácia da acupunctura na dor lombar aguda são inconclusivos. Relativamente à dor lombar crónica, a acupunctura demonstrou ser mais eficaz do que os grupos cuja intervenção foi: «sem tratamento» e «placebo». A associação da acupunctura com tratamento convencional foi mais eficaz do que o tratamento convencional isoladamente; por outro lado, a acupunctura não demonstrou ser mais eficaz quando comparada com «outros tratamentos».

A comparação directa da acupunctura com a terapêutica convencional, nomeadamente no estudo GERAC, ${ }^{1}$ um ECA que envolveu 1802 doentes com dor lombar, foi decisiva para a inclusão da acupunctura nos tratamentos comparticipados por companhias de seguros na Alemanha, equiparando-a pela primeira vez à terapêutica convencional.

Foram detectadas limitações nalguns estudos, nomeadamente em relação ao seguimento insuficiente (com avaliação apenas a curto-prazo), à falta de uniformidade quer das técnicas usadas pelos terapeutas quer da sua experiência formativa/profissional e a alguma falta de investimento na comparação da acupunctura com outras terapêuticas mais tradicionalmente usadas. Outra dificuldade existente nos ECA foi a impossibilidade de efectuar estudos duplamente cegos, já que o terapeuta sabe sempre qual a técnica que estava a aplicar, ao contrário do doente. No entanto, havia sempre o cuidado da avaliação da dor e da função ser feita por investigadores que não tinham o conhecimento acerca de qual a técnica/terapêutica usada no paciente.

A dor lombar inespecífica é um motivo de consulta extremamente frequente no âmbito da Medicina Geral e Familiar, pelo que a sua abordagem terapêutica é de enorme importância. Tendo em conta o facto de a acupunctura ser um método terapêutico cada vez mais usado no tratamento da dor crónica e, em particular, da 
dor lombar, torna-se importante perceber qual a sua verdadeira eficácia.

A revisão realizada não permitiu retirar conclusões claras acerca da eficácia da acupunctura no tratamento da dor lombar inespecífica aguda. Relativamente à dor lombar inespecífica crónica, ficou demonstrado que a acupunctura tem eficácia, quer isoladamente, quer como co-adjuvante de outros métodos terapêuticos, pelo que se atribui uma força de recomendação A. São necessários mais ECA, de longa duração e de boa qualidade metodológica, no sentido de avaliar comparativamente a acupunctura e outros métodos terapêuticos tradicionais na abordagem da dor lombar crónica, de modo a perceber se poderá haver alguma indicação específica da acupunctura, assim como em casos de dor lombar aguda, face aos dados inconclusivos apresentados nos estudos previamente realizados, para aferir a sua eficácia e indicação.

\section{REFERÊNCIAS BIBLIOGRÁFICAS}

1. Haake M, Müller HH, Schade-Brittinger $C$, Basler HD, Schäfer H, Maier $C$, et al. German Acupuncture Trials (GERAC) for chronic low back pain. Arch Intern Med 2007 Sep 24; 167 (17): 1892-8.

2. Rooney L. Acupuncture in the treatment of non-specific low back pain in an adult population: a review of the eEvidence. Internet J Adv Nurs Pract 2008; 9 (2). Available at http://www.ispub.com/journal/the_internet_journal_of_advanced_nursing_practice/volume_9_number_2_8/article/acupuncture_in_the_treatment_of_non_specific_low_ back_pain_in_an_adult_population_a_review_of_the_evidence.html [acedido em 20/05/2010].

3. Cherkin DC, Sherman KJ, Hogeboom CJ, Erro JH, Barlow WE, Deyo RA, et al. Efficacy of acupuncture for chronic low back pain: protocol for a randomized controlled trial. Trials 2008 Feb 28, 9: 10.

4. Manheimer E, White A, Berman B, Forys K, Ernst E. Meta-analysis: acupuncture for low back pain. Ann Intern Med 2005 Apr 19; 142 (8): 651-63.

5. Yuan J, Purepong N, Kerr DP, Park J, Bradbury I, McDonough S. Effectiveness of acupuncture for low back pain. Spine 2008 Nov 1; 33 (23): E887-900.

6. Witt C, Jena S, Selim D, Brinkhaus B, Reinhold T, Wruck K, et al. Pragmatic randomized trial evaluating the clinical and economic effectiveness of acupuncture for chronic low back pain. Am J Epidemiol 2006 Sep 1; 164 (5): 487-96.

7. Furlan AD, van Tulder MW, Cherkin DC, Tsukayama H, Lao L, Koes BW, et al. Acupuncture and dry-needling for low back pain. Cochrane Database Syst Rev. 2005 Jan 25; (1): CD001351.

8. Eisenberg D, Post D, Davis R, Connelly MT, Legedza AT, Hrbek AL, et al. Addition of choice of complementary therapies to usual care for acute low back pain: a randomized controlled trial. Spine 2007 Jan 15; 32 (2):
151-8.

9. Yuan J, Kerr D, Park J, Liu XH, McDonough S. Treatment regimens of acupuncture for low back pain: a systematic review. Complement Ther Med 2008 Oct; 16 (5): 295-304.

10. Kong JC, Lee MS, Shin BC. Randomized clinical trials on acupuncture in Korean literature: a systematic review. Evid Base Complement Alternat Med 2009 Mar; 6 (1): 41-8.

11. Vickers A. Acupuncture. Eff Health Care 2001; 7 (2): 1-13.

12. Zhao ZQ. Neural mechanism underlying acupuncture analgesia. Prog Neurobiol 2008; 85 (4): 355-75.

13. Weidenhammer W, Linde K, Streng A, Hoppe A. Melchart D. Acupuncture for chronic low back pain in routine care: a multicenter observational study. Clin J Pain 2007 Feb; 23 (2): 128-135.

14. Ebell MH, Siwek J, Weiss BD, Woolf SH, Susman J, Ewingman B, et al. Strength of Recommendation Taxonomy (SORT): a patient-centered approach to grading evidence in the medical literature. Am Fam Physician 2004 Feb 1; 69 (3): 548-56.

15. Keller A, Hayden J, Bombardier C, van Tulder M. Effect sizes of non-surgical treatments of non-specific low-back pain. Eur Spine J 2007 Nov; 16 (11): 1776-88.

16. Fennen BC, Esquivel RA. Acupuncture and electroacupuncture: evidence-based treatment guidelines. Calistoga, CA: Council of Acupuncture and Oriental Medicine Associates; 2004.

17. Chou R, Qaseem A, Snow V, Casey D, Cross JT Jr, Shekelle P, et al. Diagnosis and treatment of low back pain: a joint clinical practice guideline from the American College of Physicians and the American Pain Society. Ann Intern Med 2007 Oct 2; 147 (7): 478-91.

18. Early management of persistent non-specific low back pain. National Institute for Health and Clinical Excellence Clinical Guideline; 2009.

19. Inoue M, Kitakoji H, Ishizaki N, Tawa M, Ysno T, Katsumi Y, et al. Relief of low back pain immediately after acupuncture treatment: a randomised, placebo controlled trial.Acupunct Med 2006 Sep; 24 (3): 1038.

20. Itoh K, Katsumi Y, Hirota S, Kitakoji H. Effects of trigger point acupuncture on chronic low back pain in elderly patients: a sham-controlled randomised trial. Acupunct Med 2006 Mar; 24 (1): 5-12.

21. Kennedy S, Baxter GD, Kerr DP, Bradburyn I, Park J, McDonough SM. Acupuncture for acute non-specific low back pain: a pilot randomised non-penetrating sham controlled trial. Complement Ther Med 2008 Jun; 16 (3): 139-46.

22. Mayer DJ. Acupuncture: an evidence-based review of the clinical literature. Annu Rev Med 2000; 51: 49-63.

23. Chou R, Huffman LH;American Pain Society;American College of Physicians. Nonpharmacologic therapies for acute and chronic low back pain: a review of the evidence for an American Pain Society / American College of Physicians clinical practice guideline. Ann Intern Med 2007 Oct 2; 147 (7): 492-504.

24. Ammendolia C, Furlan AD, Imamura M, Irvin E, van Tulder M. Evidenceinformed management of chronic low back pain with needle acupuncture. Spine J 2008 Jan-Feb; 8 (1): 160-72.

25. Van Tulder MW, Furlan AD, Gagnier JJ. Complementary and alternative therapies for low back pain. Best Pract Res Clin Rheumatol 2005 Aug; 19 (4): 639-54. 


\section{ENDEREÇO PARA CORRESPONDÊNCIA}

João Ricardo Alves Monteiro

Rua Frei João de Vila do Conde, N. $12-2 .^{\circ}$ andar

4480-817 Vila do Conde
E-mail: jramonteiro@gmail.com

Recebido em 05/01/2010

Aceite para publicação em 30/04/2010

\section{ABSTRACT}

\section{ACUPUNCTURE IN LOW BACK PAIN: IS IT EFFECTIVE?}

Objectives: To review the available evidence on the effectiveness of acupuncture in the treatment of low back pain. Data Sources: MEDLINE, National Guideline Clearinghouse, Guidelines Finder, Cochrane Library, DARE, Bandolier, UpToDate and Index of Portuguese Medical Magazines.

Review Methods: Research using the MeSH terms acupuncture and low back pain and the DeCS terms acupuntura e dor lombar. The search was limited to articles published between January 2000 and May 2009 in english, portuguese and spanish. American Family Physician s Strength of Recommendation Taxonomy (SORT) was used to assess the level of evidence.

Results: Two meta-analysis, six systematic reviews, three randomised controlled trials and three guidelines met inclusion criteria. Guidelines say that acupuncture might be helpful in the treatment of low back pain. Unspecific chronic low back pain: the meta-analysis, systematic reviews and randomised controlled trials demonstrated that acupuncture is more effective than notreatment and that the association of acupuncture with conventional treatment is more effective than isolated conventional treatment. The studies didn $t$ demonstrate that acupuncture was more effective than placebo or conventional therapies. Acute low back pain: data are inconclusive.

Conclusions: Acupuncture is effective in the treatment of unspecific chronic low back pain, isolated or in addition to other interventions (SORT A); it was not proved that acupuncture was superior to other interventions. In the treatment of acute low back pain, there was no evidence of effectiveness of acupuncture. It would be advisable to do high quality, long-term, controlled and randomised studies, addressing its cost-benefit.

Keywords: Acupuncture; Low Back Pain. 